\title{
Changes in responsiveness to bicarbonate under capacitating conditions in liquid preserved boar spermatozoa in vitro
}

\author{
H. Henning', A.M. Petrunkina' ${ }^{1,2}$, R.A.P. Harrison ${ }^{3}$ and D. Waberski ${ }^{1}$ \\ 'Unit for Reproductive Medicine of Clinics, University of Veterinary Medicine Hannover, Hannover, \\ Germany ${ }^{2}$ Cambridge Institute for Medical Research, University of Cambridge, UK ${ }^{3} 11$ London Rd, Great \\ Shelford, Cambridge CB22 5DB, UK
}

Liquid-stored boar semen is commonly used for artificial insemination (Al) up to $72 \mathrm{~h}$ after dilution. Insemination with semen stored for longer periods generally results in reduced fertility. Standard semen parameters, i.e. motility and membrane integrity, usually give no indication of this reduction. Therefore, more sensitive methods are needed for detection of storage-induced changes in sperm quality. Capacitation has long been known to be an essential step in fertilization. In a number of studies bicarbonate has been shown to be the key capacitating agent in boar sperm in vitro (reviewed in Harrison \& Gadella 2005). The ability of sperm to respond to bicarbonate in vitro by undergoing capacitatory changes can be measured as a sperm property crucial to fertilization (Petrunkina et al. 2005a; Silva \& Gadella 2006). In this study we used calcium influx as a parameter to investigate the responsiveness of stored semen samples to bicarbonate. This parameter has been shown to be sensitive with respect to evaluating detrimental effect of cooling during liquid storage of boar sperm (Petrunkina et al. 2005b).

Three ejaculates from each of 14 boars of proven fertility were diluted in Beltsville Thawing Solution (BTS) extender to a concentration of $20 \times 10^{6}$ sperm $/ \mathrm{ml}$ and stored at $17^{\circ} \mathrm{C}$. After 12,24 , 72,120 and $168 \mathrm{~h}$ of storage, motility was assessed in the diluted semen with a CASA-system, and membrane integrity was checked with propidium iodide (PI) and FITC-conjugated peanut agglutinin (FITC-PNA) using a flow cytometer. Samples were then washed through Percoll, loaded with the calcium probe Fluo-3-AM and $\mathrm{PI}$, and incubated at $38^{\circ} \mathrm{C}$ in parallel in two variants of a Tyrode's medium. Medium A contained $15 \mathrm{mM}$ bicarbonate as well as $2 \mathrm{mM} \mathrm{Ca}^{2+}$, whereas bicarbonate was omitted from medium $\mathrm{B}$; incubation in medium $\mathrm{A}$ was performed under $5 \% \mathrm{CO}_{2}$. Changes in $\mathrm{Ca}^{2+}$ influx were assessed on a flow cytometer at $3,20,40,60,90,120,150$ and $180 \mathrm{~min}$. The resulting kinetics of cell sub-populations were compared between media and storage time points, based on analyses of the non-agglutinated population.

During storage, motility declined only from $89.0 \pm 3.2$ to $74.4 \pm 10.4 \%(p=0.001)$ and membrane integrity from $83.1 \pm 4.2$ to $71.0 \pm 20.9 \%(p-0.001)$. However, bicarbonate induced marked changes in membrane permeability, as measured by increases in the population of $\mathrm{Ca}^{2+}$-positive and PI-negative (live) cells as well as by increases in the population of PI-positive (dead) cells. After 12 and $24 \mathrm{~h}$ of storage, the population of $\mathrm{Ca}^{2+}$-positive and $\mathrm{Pl}$-negative cells reached a maximum within 90 min of incubation in medium $A$, but as storage was prolonged the increase lessened although it reached its maximum more rapidly (after $40-60 \mathrm{~min}$ ). A time point of 60 min was chosen for comparisons between storage periods and media. Values for the total $\% \mathrm{Ca}^{2+}$-positive / PI-negative cells in medium A declined significantly from $21.6 \pm 6.4 \%$ at $12 \mathrm{~h}$ to $15.7 \pm 2.78 \%$ at $72 \mathrm{~h}$ of storage $(p<0.01)$ after which they stayed at a constant level. At 3 min of incubation proportions of PI-positive (dead) cells in medium A varied between $9.8 \pm 4.9 \%$ at $12 \mathrm{~h}$ of storage and $14.7 \pm 3.3 \%$ at $168 \mathrm{~h}$, whereas after $60 \mathrm{~min}$ of incubation their percentage had risen to around $46 \%$ regardless of storage period. In contrast, in medium B, 60 min values of both populations (i.e. $\mathrm{Ca}^{2+}$-positive / PI-negative and PI-positive cells), though initially much lower than in Medium A, increased significantly through- 
out the whole storage period $(p<0.01)$ until they were comparable with the values in medium $A$. Response to capacitating conditions as measured by the change in $\% \mathrm{Ca}^{2+}$-negative / $\mathrm{Pl}$-negative cells between $3 \mathrm{~min}$ and $60 \mathrm{~min}$ of incubation $\left(\Delta_{60-3}\right)$ declined significantly in medium $A$ during storage from $58.6 \pm 8.2 \%$ after $12 h$ to $36.4 \pm 8.6 \%$ after $168 h(p<0.001)$, whereas in medium $B$ it increased from $6.8 \pm 4.4 \%$ to $24.0 \pm 3.7 \%(p=0.001)$. However, storage also resulted in a change in the sub-population distributions at the initial $(3 \mathrm{~min})$ incubation point. There were significantly higher $(p<0.01)$ percentages of $\mathrm{Ca}^{2+}$-positive / Pl-negative cells and PI-positive cells detected in both medium $A$ and medium $B$ : in medium A $10.1 \% \mathrm{Ca}^{2+}$-positive/PI-negative and $14.7 \%$ Pl-positive after $168 \mathrm{~h}$ versus $3.3 \%$ and $9.8 \%$ respectively after $12 \mathrm{~h}$; in medium $B$ 9.1\% Ca $\mathrm{Ca}^{2+}$-positive / Pl-negative and $14.7 \% \mathrm{Pl}$-positive after $168 \mathrm{~h}$ versus $2.1 \%$ and $7.2 \%$ respectively after $12 \mathrm{~h}$. These data could be interpreted as indicating that storage has two effects. There was on the one hand a destabilization of the greater proportion of the population such that incubation even in the absence of bicarbonate caused membrane deterioration while incubation with bicarbonate caused such rapid membrane destabilization that the $\mathrm{Ca}^{2+}$-positive / PI-negative state was increasingly short-lived before PI entered. These findings are in agreement with those of Petrunkina et al. (2005b), reporting that levels of $\mathrm{Ca}^{2+}$ uptake at the beginning of incubation under capacitating conditions are influenced by storage conditions. However, we also obtained evidence that the cohorts of cells that were less immediately responsive to bicarbonate became refractory, reducing the overall bicarbonate response in terms of appearance of $\mathrm{Ca}^{2+}$-positive / Pl-negative plus Pl-positive cells.

As expected, motility and membrane integrity of the stored cells did not sufficiently reflect storagedependent changes in sperm quality during prolonged storage. A recent review (Petrunkina et al. 2007) has proposed several requirements for the assessment of functional sperm parameters under capacitating conditions. In accordance with these proposals, we used a kinetic experimental approach with well-defined test and control media, considering equally both initial response and subsequent kinetics. Specific sperm response to bicarbonate as measured by intracellular increases in $\mathrm{Ca}^{2+}$ in live cells declined remarkably already after $72 \mathrm{~h}$ of liquid storage and dropped to almost zero in samples stored for $168 \mathrm{~h}$. However, bicarbonate routinely caused large increases in dead cells within 20 min of incubation. In their original paper on fluo-3 detection of bicarbonate-mediated changes in boar sperm, Harrison et al. (1993) interpreted fluo-3-detectable $\mathrm{Ca}^{2+}$ entry as indicating the onset of a membrane destabilization eventually leading to cell death. Harrison (1996) proposed that if such destabilization were too rapid, fertilization would be compromised. Our findings suggest that boar semen contains a population of cells initially responsive to bicarbonate which becomes increasingly intrinsically unstable during storage and responds to bicarbonate too rapidly. In addition, more stable cohorts of cells exist that during storage become less responsive to bicarbonate. We suspect that the dual opposing effects of storage, namely destabilization and stabilization, may imply that after normal insemination life-time of most sperm in the female tract will be insufficient to ensure satisfactory fertilization levels, while the remainder may not respond to fertilizing conditions at all.

This work was supported by Development Association for Biotechnology Research (FBF e.V., Bonn)

\section{References}

Harrison RAP, Mairet B \& Miller NG 1993 Flow cytometric studies of bicarbonate-mediated $\mathrm{Ca} 2+$ influx in boar sperm populations. Molecular Reproduction \& Development 35 197-208.

Harrison RAP 1996 Capacitation mechanisms, and the role of capacitation as seen in eutherian mammals. Reproduction, Fertility \& Development 8 581-594.

Harrison RAP \& Gadella BM 2005 Bicarbonate-induced membrane processing in sperm capacitation. Theriogenology 63 342-351.

Petrunkina AM, Volker G, Brandt H, Töpfer-Petersen E \& Waberski D 2005a Functional significance of responsiveness to capacitating conditions in boar spermatozoa. Theriogenology 64 1766-1782.

Petrunkina AM, Volker $G$, Weitze KF, Beyerbach $M$, Töpfer-Petersen E \& Waberski D 2005b Detection of cooling-induced membrane changes in the response of boar sperm to capacitating conditions. Theriogenology 63 2278-2299.

Petrunkina AM, Waberski D, Günzel-Apel AR \& TöpferPetersen E 2007 Determinants of sperm quality and fertility in domestic species. Reproduction 134 3-17.

Silva PF \& Gadella BM 2006 Detection of damage in mammalian sperm cells. Theriogenology 65 958-978. 